\title{
Articular Eminence Morphology of American Historic and Contemporary Populations
}

\section{Morfologija zglobne kvržice američkih povijesnih i suvremenih populacija}

\author{
${ }^{1}$ School of Dental Medicine, University of Zagreb, 10000 Zagreb, Croatia \\ Stomatološki fakultet Sveučilišta u Zagrebu, Hrvatska \\ 2 Clinical Hospital “Dubrava”, 10000 Zagreb, Croatia \\ Klinička bolnica Dubrava, Zagreb, Hrvatska \\ ${ }^{3}$ Smithsonian Institution, Washington, DC 20002, USA \\ Institut Smithsonian, Washington, DC 20002, SAD
}

\section{Abstract}

Objectives: This study was performed to determine the values of the articular eminence inclination (AEI), the articular eminence height (AEH), and the length of the articular eminence curved line (AEL) among American prehistoric and contemporary populations. Materials and methods: The study was carried out on 120 human dry skulls divided into 4 groups: Illinois group (IP), Kentucky group (KP), African Americans group (AAP) and American Caucasians group (ACP). Each group comprised 30 human dry skulls. Measurements of AEI (by two methods), AEH and AEL were performed using computer software on 5 sagittal sections through silicone molds of the articular eminence. The obtained results were statistically analyzed at significance level of $p<0.05$. Results: No statistically significant differences of AEI, AEH and AEL values were obtained among American populations (IP, KP, AAP, ACP) neither by body side, sex and age $(p>0.05)$. The mean AEI M1 values ranged from $31.56^{\circ}$ to $38.72^{\circ}$, the mean AEI M2 values ranged from $44.14^{\circ}$ to $58.37^{\circ}$, the mean AEH ranged from 5.82 to $6.86 \mathrm{~mm}$, and the mean AEL values ranged from 11.57 to $12.73 \mathrm{~mm}$. Conclusions: AEI, AEH, AEL values are variables, but they did not differ significantly among American populations. Left-right differences as the result of natural body asymmetry were not statistically significant. Sex and age did not significantly affect the articular eminence morphology.

\author{
Received: April 10, 2021 \\ Accepted: June 21, 2021 \\ Address for correspondence \\ Josip Kranjcic \\ Department of Prosthodontics, \\ Clinical Hospital ‘Dubrava’, Avenija G. \\ Suska 6, 10000 Zagreb, Croatia \\ Telephone: +3851290 2724; Fax \\ number: +3851 2864248 \\ kranjcic@sfzg.hr \\ MeSH terms: Temporomandibular \\ Joint Disc; Population Characteristics; \\ African Americans; American Natives; \\ European Continental Ancestry Group \\ Author keywords: Skull; Temporoman- \\ dibular joint; North America; Population
}

\section{Introduction}

The temporomandibular joint (TMJ) as an integrative component of the masticatory system allows a large range of mandibular movements (1). The range of mandibular movements is dependent on the articular eminence morphology which is an important TMJ biomechanical element. During mandibular movements, the mandibular condyle and articular disc slide together (condyle-disc complex) along the posterior wall of the articular eminence (1-5).

The TMJ morphology has been investigated on primates, laboratory animals and humans correlate its morphology with biomechanics, type of consumed food, and masticatory stress (6-13). The articular eminence morphology undergoes remodeling throughout an individual's life $(14,15)$ and its morphology is affected more by function than by skull base characteristics or genetics (1). At birth, the articular eminence is completely flat (1). The full articular eminence inclination (AEI) value is reached by the age of 30 years (1, $14,16)$. The AEI values usually vary from $21^{\circ}$ to $64^{\circ}(17)$ in
Uvod

Temporomandibularni zglob (TMJ) kao dio žvačnoga sustava omogućuje donjoj čeljusti velik raspon kretnji (1). Na to utječe morfologija zglobne kvržice koja je, pak, važan biomehanički element. Tijekom kretnji donje čeljusti, kondil i zglobna pločica zajedno klize (kondil - disk kompleks) po stražnjem zidu zglobne kvržice $(1-5)$.

Morfologija TMJ-a istraživana je na primatima, laboratorijskim životinjama i ljudima te se pritom povezivala $s$ biomehanikom, vrstom konzumirane hrane i žvačnim opterećenjem (6 -13). Morfologija zglobne kvržice podložna je promjenama tijekom cijeloga života $(14,15)$ i više je pod utjecajem funkcije nego što je povezana s karakteristikama baze lubanje i genetikom (1). Pri rođenju je zglobna kvržica potpuno ravna (1). Puni nagib stražnjega zida zglobne kvržice (AEI) dostiže se s 30 godina $(1,14,16)$. AEI vrijednosti obično variraju od $21^{\circ}$ do $64^{\circ}(17)$ mjereno u odnosu na frankfurtsku horizontalu $(17,18)$. Tijekom razvoja i rasta zglobne kvržice njezina visina (AEH) i duljina zakrivljene linije od 
relation to the Frankfurt horizontal plane $(17,18)$. During the articular eminence development and growth, the articular eminence height $(\mathrm{AEH})$ and the length of articular eminence curved line (AEL) values also increase, reaching the average $\mathrm{AEH}$ value of $6 \mathrm{~mm}$, and an average AEL value of $12 \mathrm{~mm}$ (3). Furthermore, the articular eminence morphology depends on many other factors such as sex and ancestry, changes in dentition, degenerative changes of articular eminence and condyle as well as on morphological changes of the cranial base, which has been documented in the human evolutionary process (19-26).

The aim of this study was to establish the range of the AEI, AEH and the AEL values of skulls in four population groups from different periods of American history, and to determine differences, if existed, in the articular eminence morphology among these groups. One sample was from prehistoric Native American populations (Woodland Period Illinois (IP) $900 \mathrm{AD}$ to $1500 \mathrm{AD}$ ), one sample from Archaic Period Kentucky (KP) (500 BC to $500 \mathrm{AD}$ ), and two samples of early 20th century Americans (African Americans (AAP), and American Caucasians (ACP)) from Medical School cadavers at Washington University, St. Louis, Missouri. A null hypothesis was established as follows: there is no statistically significant difference in AEI, AEH and AEL values between IP, KP, AAP and ACP groups.

\section{Materials and methods}

The study was carried out on 120 human dry skulls divided into 4 groups: Woodland Period llinois population (IP; from $900 \mathrm{AD}$ to $1500 \mathrm{AD}$ years); Archaic Period Kentucky population (KP; from $500 \mathrm{BC}$ to $500 \mathrm{AD}$ years), African Americans (AAP; 20th century St. Louis, Missouri) and American Caucasian (ACP; 20th century St. Louis, Missouri). Each group comprised 30 human dry skulls. The skulls are curated at the Department of Anthropology, Smithsonian Institution, Washington DC, USA. All selected skulls were fully preserved without observable taphonomic damage in the measured area (articular eminence, fossa articularis, orbitae, and meatus acusticus externus) or/ and without visible pathological conditions in the articular eminence and glenoid fossa.

Sex was identified by biological metric and non-metric traits (pelvic shape, articular joint size, cranioscopic features) on the Illinois and Kentucky archaeological samples, and for the Terry Collection from cadavers at Washington University Medical School anatomy laboratory. The reported age (age at death) was available for AAP and ACP skulls. Because there were only two samples with known age, the skulls were grouped into three age groups: first group - individuals who were 30 years of age or younger; second group - individuals from 31 to 45 years of age; third group - individuals who were 46 years of age or older.

The investigated TMJ parameters were: the articular eminence inclination (AEI), the articular eminence height (AEH) and the length of articular eminence curved line (AEL) in the sagittal plane. The AEI was measured in degrees by two methods. Angle (Figure 1) between the line connecting the najviše do najniže točke (AEL) također rastu te dosežu prosječno AEH vrijednost od $6 \mathrm{~mm}$ i AEL vrijednost od $12 \mathrm{~mm}$ (3). Nadalje, morfologija zglobne kvržice ovisi i o mnogim drugim čimbenicima poput spola i podrijetla, promjena na zubima, degenerativnih promjena na zglobnoj kvržici i kondilu te morfoloških promjena na kranijalnoj bazi, a dokumentirane su i evolucijske promjene $(19-26)$.

Svrha istraživanja bila je utvrditi raspon AEI, AEH i AEL vrijednosti na lubanjama četiriju populacijskih skupina iz različitih razdoblja američke povijesti te ustanoviti razlike (ako postoje) u morfologiji njihovih zglobnih kvržica. U istraživanje su bili uključeni: uzorak pretpovijesne američke populacije s područja Illinoisa (IP) od 900. do 1500. godine poslije Krista, uzorak s područja Kentuckyja (KP) od 500. godine prije Krista do 500. godine poslije Krista te dva uzorka lubanja iz ranoga 20. stoljeća - američkih crnaca (AAP) i američkih bijelaca (ACP) s Medicinskog fakulteta Sveučilišta Washington u St. Louisu, Missouri. Postavljena je nulta hipoteza da nema statistički značajnih razlika u AEI, AEH i AEL vrijednostima između skupina IP, KP, AAP i ACP.

\section{Materijal i metode}

Istraživanje je provedeno na 120 ljudskih lubanja podijeljenih u četiri skupine: populacija s područja Illinoisa (IP; od 900. do 1500. god. pos. Kr.); s područja Kentuckyja (KP; od 500. god. pr. Kr. do 500. god. pos. Kr.), američki crnci (AAP; 20. , St. Louis, Missouri) i američki bijelci (ACP; 20. st., St. Louis, Missouri). U svakoj je bilo po 30 lubanja. Pohranjene su bile u Zavodu za antropologiju Instituta Smithsonian $\mathrm{u}$ Washingtonu $(\mathrm{SAD})$. Lubanje uključene u istraživanje bile su bez vidljivih tafonomskih oštećenja u području važnom za istraživanje (zglobna kvržica, zglobna jamica, orbita i meatus acusticus externus) ili bez vidljivih patoloških promjena zglobne kvržice i jamice.

Spol je određen biološkim metričkim i nemetričkim obilježjima (oblik zdjelice, veličina zglobne površine, kranioskopska obilježja) na arheološkom uzorku s područja Illinoisa i Kentuckyja te na uzorcima iz Terryjeve kolekcije iz Anatomskog laboratorija Medicinskog fakulteta Sveučilišta Washington. Dob (u trenutku smrti) bila je poznata za AAP i ACP uzorak. Dakle, samo u dvjema skupinama bila je poznata dob te su te lubanje podijeljene $\mathrm{u}$ tri dobne skupine: prva skupina - 30 godina ili mlađi; druga skupina - uzorci od 31. do 45. godine; treća skupina - uzorci od 46. godine ili stariji.

Istraživani parametri bili su: nagib stražnjega zida zglobne kvržice (AEI), visina zglobne kvržice (AEH) i duljina zakrivljene linije od najviše do najniže točke na zglobnoj kvržici (AEL) u sagitalnoj ravnini. AEI je mjeren u stupnjevima dvjema metodama. Kut (slika 1.) između linije koja spaja najvišu točku zglobne jamice $s$ najnižom točkom zglobne kvržice u odnosu prema frankfurtskoj horizontali prikazuje metoda 1 (M1); kut (slika 2.) između linije koja je najbolje prilagođe- 

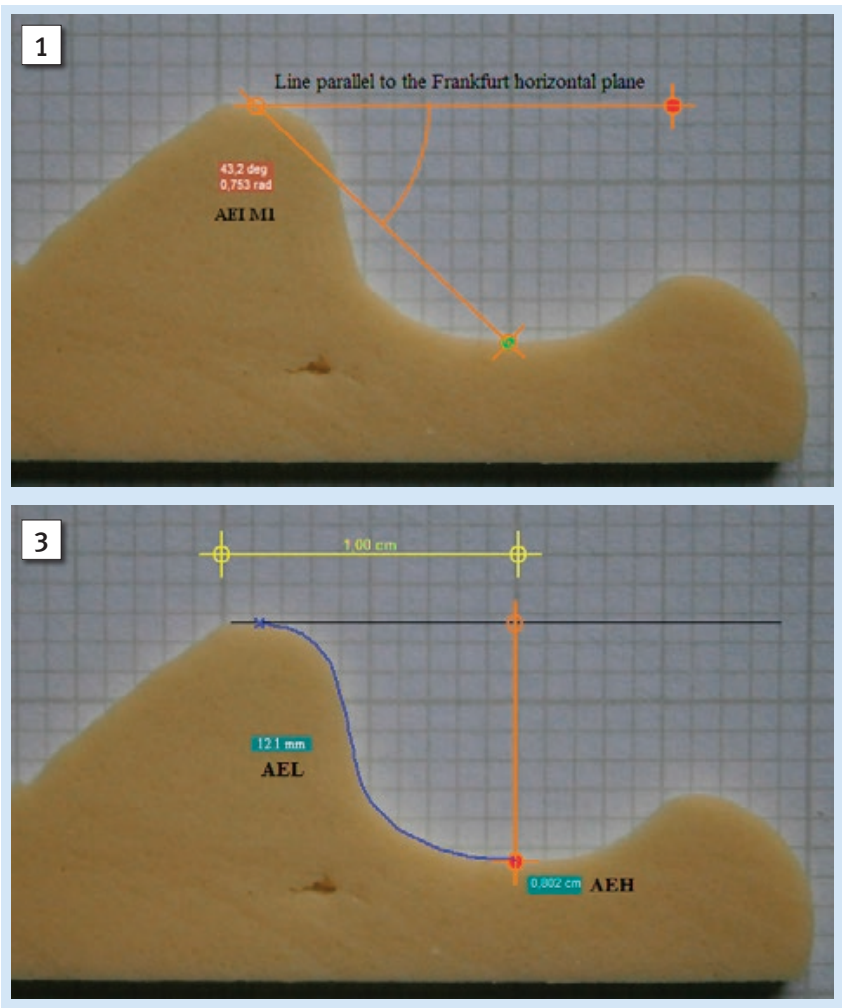

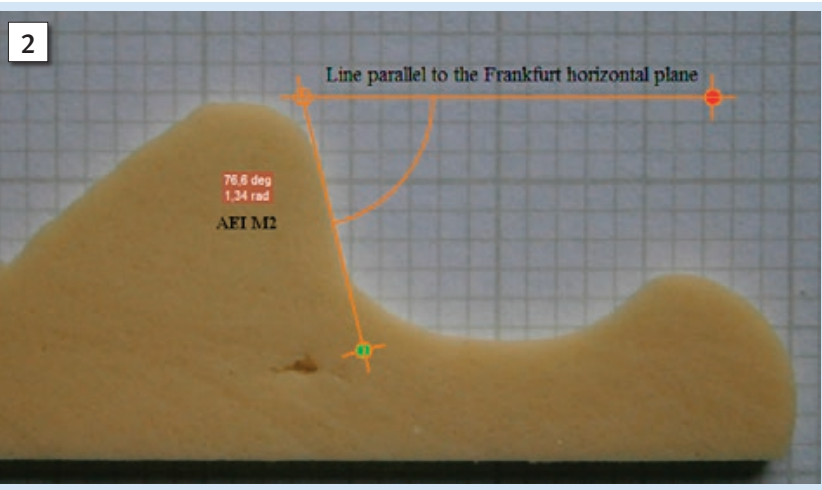

Figure 1 Measurement of articular eminence inclination (AEI) by method M1.

Slika 1. Mjerenje nagiba stražnjega zida zglobne kvržice (AEI) metodom $M 1$

Figure 2 Measurement of articular eminence inclination (AEI) by method M2.

Slika 2. Mjerenje nagiba stražnjega zida zglobne kvržice (AEI) metodom M2

Figure 3 Measurement of articular eminence height (AEH) and length of articular eminence curved line (AEL).

Slika 3. Mjerenje visine zglobne kvržice (AEH) i duljine zakrivljene linije od najviše do najniže točke na zglobnoj kvržici (AEL) most superior point of the glenoid fossa and the most inferior point of the articular eminence with the Frankfurt horizontal plane presented method 1 (M1); while the angle (Figure 2) between the best fitting line to the posterior wall of the articular eminence and the Frankfurt horizontal plane presented method 2 (M2). The AEH was measured as vertical distance $(\mathrm{mm})$ between the most superior and the most inferior point of the articular eminence, while the AEL ( $\mathrm{mm}$ ) was measured as length of curved line between the most superior point of the glenoid fossa and the most inferior point of the articular eminence (Figure 3). The AEI, AEH and AEL were measured on five digitalized sagittally positioned sections through the left and right side silicone impressions of the articular eminence and glenoid fossa. Finally, the measured AEI, AEH and AEL values were presented as means of the values measured on all five sections through silicone impressions of articular eminence and glenoid fossa.

Silicone impressions (Optosil, Heraeus, Hanau, Germany) of left and right side articular eminence were made for each skull. First, the axio-quick face bow (SAM Präzisionstechnik GmbH, Munich, Germany) was mounted on each skull with ear sets inserted into the meatus acusticus externus and the nasion extension placed on nasion anatomical point in order to achieve parallelism of the face bow with the skull's Frankfurt horizontal plane. In order to achieve a parallelism of the silicone impression base with the Frankfurt horizontal plane, a specially designed device was used. When this device was placed on the face bow, its horizontal plate was parallel to the Frankfurt horizontal plane. After application of the impression material in the area of articular eminence and glenoid fossa, the horizontal plate of specially designed device was slightly pressed over the impression material in or- na stražnjemu zidu zglobne kvržice i frankfurtske horizontale određen je kao metoda 2 (M2). AEH je mjeren kao vertikalna udaljenost $(\mathrm{mm})$ između najviše i najniže točke zglobne kvržice, a AEL (mm) kao duljina zakrivljene linije između najviše točke zglobne jamice i najniže točke zglobne kvržice (slika 3.). AEI, AEH i AEL mjereni su na pet digitaliziranih sagitalnih presjeka kroz lijevi i desni silikonski otisak zglobne kvržice i jamice. Konačno, izmjerene AEI, AEH i AEL vrijednosti prikazane su kao srednje vrijednosti svih pet rezova kroz silikonske otiske zglobne kvržice i jamice.

Za svaku lubanju napravljen je silikonski otisak (Optosil, Heraeus, Hanau, Njemačka) zglobne kvržice lijeve i desne strane. Najprije je na svaku lubanju postavljen obrazni luk (SAM Präzisionstechnik GmbH, München, Njemačka) tako da su nastavci za uške postavljeni u vanjski slušni hodnik, a nastavak za nasion postavljen je na nasion-anatomsku točku da bi se osigurala paralelnost obraznoga luka s frankfurtskom horizontalom. Da bi se osigurala paralelnost baze silikonskog otiska s frankfurtskom horizontalom, primijenjena je posebno oblikovana sprava. Nakon što je postavljena na obrazni luk, njezina horizontalna ploča bila je paralelna s frankfurtskom horizontalom. Nakon što je otisni materijal postavljen u područje zglobne kvržice i jamice, horizontalna ploča dizajnirane sprave lagano se pritisne preko otisnoga materijala da bi se postigla paralelnost baze silikonskog otiska i frankfurtske horizontale. Poslije stvrdnjavanja materijala silikonski otisci uklonjeni su s lubanje. Silikonski otisci razrezani su [u anteroposteriornom smjeru (sagitalna ravnina)] od lateralne prema medijalnoj površini u pet rezova (između svakoga reza udaljenost od $4 \mathrm{~mm}$ ) primjenom jajoreza (Westmark Gmbh, Lennestadt-Elspe, Njemačka). Prvi, najlateralniji rez učinjen je kroz najlateralniji vrh zglobne kvržice (tuberculum articula- 
der to form a silicone impression base parallel to the Frankfurt horizontal plane. After the silicone impression material was cured, the impressions of the articular eminence and glenoid fossa were removed from the skulls. The silicone impressions were sliced (in anteroposterior direction (sagittal plane)) from lateral to medial side into five sections (between each section was a distance of $4 \mathrm{~mm}$ ) using common egg slicer (Westmark Gmbh, Lennestadt-Elspe, Germany). First the most lateral section was made through the most lateral top of the articular eminence (articular tubercle). Each section was placed on the graph paper on the horizontal surface of the camera holder (Hama, Manheim, Germany) and digitalized by Olympus C-770 camera (Olympus, Tokyo, Japan) with a distance of $35 \mathrm{~cm}$. The AEI M1, AEI M2, AEH and AEL were measured using the computer software VistaMetrix (Skillcrest LLC, Tucson, USA).

Data analysis was performed using SPSS 15.0 statistical software (SPSS Inc., Chicago, IL, USA) and the results were obtained with descriptive statistics, the independent-sample Student's t-test, one way ANOVA, and the Pearson correlation. The results were considered significant at the $\mathrm{p}<0.05$ level.

\section{Results}

Distribution according to sex: the IP group - $12(40 \%)$ males and $18(60 \%)$ females, the KP group - $20(66.7 \%)$ males and $10(33.3 \%)$ females, the AAP group - 15 (50\%) males and $15(50 \%)$ females, ACP group - $15(50 \%)$ males and $15(50 \%)$ females.

Age at death was known only for AAP and ACP skulls. The individuals in AAP and ACP groups were divided into 3 age groups: 30 years of age or younger - $10(33.3 \%)$ for the AAP, and $6(20 \%)$ for the ACP skulls; 31 to 45 years of age $10(33.3 \%)$ for the AAP, and $14(46.7 \%)$ for the ACP skulls; and 46 years of age or older - $10(33.3 \%)$ for AAP skulls, and 10 (33.3\%) for the ACP skulls.

Mean AEI M1 values for all five sections through the silicone impressions of articular eminence are presented in Table 1.; mean AEI M2 values for all five sections through the silicone impressions of articular eminence are presented in $\mathrm{Ta}$ ble 2.; mean AEH values for all five sections through the sili- re). Svaki rez postavljen je na milimetarski papir na horizontalnoj površini držača kamere (Hama, Manheim, Njemačka) i digitaliziran fotoaparatom Olympus C-770 (Olympus, Tokio, Japan) s udaljenosti od $35 \mathrm{~cm}$. Zatim su AEI M1, AEI M2, AEH i AEL mjereni u računalnom programu VistaMetrix (Skillcrest LLC, Tucson, SAD).

Analiza podataka učinjena je u SPSS 15.0 statističkom programu (SPSS Inc., Chicago, IL, SAD), a rezultati su obrađeni metodom deskriptivne statistike, nezavisnim studentovim t-testom, jednosmjernom analizom varijance ANOVA i Pearsonovom korelacijom. Rezultati su obrađeni na razini značajnosti $\mathrm{p}<0,05$.

\section{Rezultati}

Raspodjela lubanja s obzirom na spol: IP skupina - 12 muških (40 \%) i 18 ženskih (60 \%); KP skupina - 20 muških $(66,7 \%)$ i 10 ženskih (33,3\%); AAP skupina - 15 muških $(50 \%)$ i 15 ženskih (50\%); ACP skupina - 15 muških (50\%) i 15 ženskih (50\%).

Dob u trenutku smrti bila je poznata samo za lubanje iz skupina AAP i ACP. Uzorci iz skupina AAP i ACP bili su podijeljeni u tri dobne skupine: 30 godina ili mladi - 10 iz skupine AAP $(33,3 \%)$ i 6 , iz ACP skupine (20\%); od 31. do 45. godine - 10 iz AAP-a $(33,3 \%)$ i 14 iz ACP-a (46,7 \%); 46 godina ili stariji - 10 iz AAP-a (33,3\%) i 10 iz ACP-a (33,3\%).

Srednje AEI M1 vrijednosti svih pet rezova kroz silikonske otiske zglobne kvržice prikazane su u tablici 1.; srednje AEI M2 vrijednosti svih pet rezova kroz silikonske otiske zglobne kvržice prikazane su u tablici 2.; srednje AEH vrijednosti svih pet rezova kroz silikonske otiske zglobne kvrži-

Table 1 AEI M1 mean values (of all five sections through silicone impressions) for each group according to the body side and sex.

Tablica 1. AEI M1srednje vrijednosti (za svih 5 rezova kroz silikonske otiske) za svaku skupinu s obzirom na stranu tijela i spol

\begin{tabular}{|c|c|c|c|c|c|c|c|c|c|c|c|c|}
\hline \multirow{2}{*}{\multicolumn{2}{|c|}{$\begin{array}{c}\text { AEI M1 } \\
\left({ }^{\circ}\right)\end{array}$}} & \multirow{2}{*}{$\mathbf{N}$} & \multirow{2}{*}{ Min } & \multirow{2}{*}{$\begin{array}{l}\text { Max } \\
\text { Maks. }\end{array}$} & \multirow{2}{*}{$\mathbf{X}$} & \multirow{2}{*}{ SD } & \multicolumn{3}{|c|}{ Men • Muški } & \multicolumn{3}{|c|}{ Women • Ženski } \\
\hline & & & & & & & $\mathbf{N}$ & $\mathbf{X}$ & SD & $\mathbf{N}$ & $\mathrm{X}$ & SD \\
\hline Illinois & $\mathrm{R}$ & 30 & 21.32 & 40.28 & $31.56^{1}$ & 4.75 & 12 & 32.74 & 2.79 & 18 & 31.18 & 5.58 \\
\hline \multirow{2}{*}{ Kentucky } & $\mathrm{R}$ & 30 & 23.96 & 43.52 & 34.36 & 4.59 & 20 & 33.87 & 5.12 & 10 & 35.35 & 3.33 \\
\hline & $\mathrm{L}$ & 30 & 20.66 & 44.34 & 34.54 & 5.75 & 20 & 33.94 & 6.11 & 10 & 35.74 & 5.04 \\
\hline \multirow{2}{*}{$\begin{array}{l}\text { Africans Americans } \\
\text { Američki crnci }\end{array}$} & $\mathrm{R}$ & 30 & 22.16 & 45.36 & 36.48 & 5.68 & 15 & 37.83 & 6.43 & 15 & 35.13 & 4.63 \\
\hline & $\mathrm{L}$ & 30 & 24.72 & 45.84 & 36.42 & 5.65 & 15 & 37.79 & 5.55 & 15 & 35.04 & 5.60 \\
\hline \multirow{2}{*}{$\begin{array}{l}\text { American Caucasians } \\
\text { Američki bijelci }\end{array}$} & $\mathrm{R}$ & 30 & 23.96 & 47.64 & 36.26 & 6.42 & 15 & $38.68^{a}$ & 5.73 & 15 & $33.84^{a}$ & 6.33 \\
\hline & $\mathrm{L}$ & 30 & 26.64 & 49.46 & 38.72 & 5.57 & 15 & 40.59 & 5.09 & 15 & 36.85 & 5.56 \\
\hline
\end{tabular}

AEI M1 - articular eminence inclination measured by method $1 \bullet$ nagib stražnjega zida zglobne kvržice izmjeren metodom 1 ; N - number of skulls $\bullet$ broj lubanja; Min - minimal value $\bullet$ najmanja vrijednost; Max - maximal value $\bullet$ najveća vrijednost; X - mean value $\bullet$ srednja vrijednost; SD - standard deviation • standardna devijacija; R - right $\bullet$ desno; L - left $\bullet$ lijevo; ${ }^{1}$ - statistically significant difference between $\mathrm{R}$ and $\mathrm{L}$ samples, $\mathrm{p}<0.05 \bullet$ statistički značajna razlika između $\mathrm{R}$ i L uzoraka, $\mathrm{p}<0,05$; ${ }^{a}$ - statistically significant difference according to the sex, $\mathrm{p}<0.05 \bullet$ statistički značajna razlika s obzirom na spol, $\mathrm{p}<0,05$ 
Table 2 AEI M2 mean values (of all five sections through silicone impressions) for each group according to the body side and sex.

Tablica 2. AEI M2 srednje vrijednosti (za svih 5 rezova kroz silikonske otiske) za svaku skupinu s obzirom na stranu tijela i spol

\begin{tabular}{|c|c|c|c|c|c|c|c|c|c|c|c|c|}
\hline \multirow{2}{*}{\multicolumn{2}{|c|}{$\begin{array}{c}\text { AEI M2 } \\
\left({ }^{\circ}\right)\end{array}$}} & \multirow{3}{*}{$\begin{array}{l}\mathbf{N} \\
30\end{array}$} & \multirow{3}{*}{$\begin{array}{l}\text { Min } \\
30.74\end{array}$} & \multirow{3}{*}{$\begin{array}{c}\text { Max • } \\
\text { Maks. } \\
55.60\end{array}$} & \multirow{3}{*}{$\begin{array}{c}\mathbf{X} \\
44.14^{1}\end{array}$} & \multirow{3}{*}{$\begin{array}{l}\text { SD } \\
6.84\end{array}$} & \multicolumn{3}{|c|}{ Men • Muški } & \multicolumn{3}{|c|}{ Women • Ženski } \\
\hline & & & & & & & \multirow{2}{*}{$\begin{array}{l}\mathbf{N} \\
12 \\
\end{array}$} & \multirow{2}{*}{$\begin{array}{c}\mathbf{X} \\
43.41 \\
\end{array}$} & \multirow{2}{*}{$\begin{array}{l}\text { SD } \\
4.50\end{array}$} & \multirow{2}{*}{$\begin{array}{l}\mathbf{N} \\
18\end{array}$} & \multirow{2}{*}{$\begin{array}{c}\mathrm{X} \\
44.63 \\
\end{array}$} & \multirow{2}{*}{$\begin{array}{c}\text { SD } \\
8.13\end{array}$} \\
\hline Illinois & $\mathrm{R}$ & & & & & & & & & & & \\
\hline 11171001s & $\mathrm{L}$ & 30 & 37.98 & 63.46 & $52.67^{1}$ & 6.62 & 12 & 54.04 & 5.70 & 18 & 51.76 & 7.18 \\
\hline \multirow{2}{*}{ Kentucky } & $\mathrm{R}$ & 30 & 31.42 & 60.16 & 46.44 & 7.72 & 20 & 44.99 & 8.17 & 10 & 49.36 & 6.09 \\
\hline & $\mathrm{L}$ & 30 & 28.16 & 68.54 & 50.25 & 10.12 & 20 & 47.87 & 10.19 & 10 & 55.00 & 8.56 \\
\hline \multirow{2}{*}{$\begin{array}{l}\text { Africans Americans } \\
\text { Američki crnci }\end{array}$} & $\mathrm{R}$ & 30 & 28.16 & 68.26 & 50.24 & 9.77 & 15 & 53.20 & 10.77 & 15 & 47.28 & 7.94 \\
\hline & $\mathrm{L}$ & 30 & 33.60 & 74.82 & 52.50 & 10.29 & 15 & $56.38^{\mathrm{a}}$ & 10.04 & 15 & $48.62^{\mathrm{a}}$ & 9.29 \\
\hline \multirow{2}{*}{$\begin{array}{l}\text { American Caucasians } \\
\text { Američki bijelci }\end{array}$} & $\mathrm{R}$ & 30 & 31.18 & 73.42 & 52.85 & 12.06 & 15 & 56.98 & 11.06 & 15 & 48.72 & 11.93 \\
\hline & $\mathrm{L}$ & 30 & 33.44 & 79.62 & 58.37 & 10.37 & 15 & 61.11 & 9.08 & 15 & 55.63 & 11.15 \\
\hline
\end{tabular}

AEI M2 - articular eminence inclination measured by method $2 \bullet$ nagib stražnjega zida zglobne kvržice izmjeren metodom $1 ; \mathrm{N}$ - number of skulls $\bullet$ broj lubanja; Min - minimal value $\bullet$ najmanja vrijednost; Max - maximal value $\bullet$ najveća vrijednost; X - mean value $\bullet$ srednja vrijednost; $S D$ - standard deviation • standardna devijacija; R - right $\bullet$ desno; L - left $\bullet$ lijevo; ${ }^{1}$ - statistically significant difference between $\mathrm{R}$ and $\mathrm{L}$ samples, $\mathrm{p}<0.05 \bullet$ statistickki značajna razlika između $\mathrm{R}$ i L uzoraka, $\mathrm{p}<0,05$; $^{\mathrm{a}}$ - statistically significant difference according to the sex, $\mathrm{p}<0.05 \bullet$ statistički značajna razlika s obzirom na spol, $\mathrm{p}<0,05$

Table 3 AEH mean values (of all five sections through silicone impressions) for each group according to the body side and sex.

Tablica 3. AEH srednje vrijednosti (za svih 5 rezova kroz silikonske otiske) za svaku skupinu s obzirom na stranu tijela i spol

\begin{tabular}{|c|c|c|c|c|c|c|c|c|c|c|c|c|}
\hline \multirow{2}{*}{\multicolumn{2}{|c|}{$\begin{array}{c}\text { AEH } \\
(\mathrm{mm})\end{array}$}} & \multirow{3}{*}{$\begin{array}{l}\mathbf{N} \\
30 \\
\end{array}$} & \multirow{3}{*}{$\begin{array}{l}\text { Min } \\
4.10 \\
\end{array}$} & \multirow{3}{*}{$\begin{array}{c}\text { Max • } \\
\text { Maks. } \\
7.59\end{array}$} & \multirow{3}{*}{$\begin{array}{c}\mathbf{X} \\
5.82\end{array}$} & \multirow{3}{*}{$\begin{array}{l}\text { SD } \\
1.06\end{array}$} & \multicolumn{3}{|c|}{ Men • Muški } & \multicolumn{3}{|c|}{ Women • Ženski } \\
\hline & & & & & & & \multirow{2}{*}{$\begin{array}{l}\mathbf{N} \\
12\end{array}$} & \multirow{2}{*}{$\frac{\mathbf{X}}{6.24}$} & \multirow{2}{*}{$\begin{array}{c}\text { SD } \\
0.90\end{array}$} & \multirow{2}{*}{$\begin{array}{l}\mathbf{N} \\
18 \\
\end{array}$} & \multirow{2}{*}{$\begin{array}{c}\mathbf{X} \\
5.54\end{array}$} & \multirow{2}{*}{$\begin{array}{l}\text { SD } \\
1.09\end{array}$} \\
\hline & $\mathrm{R}$ & & & & & & & & & & & \\
\hline Illinois & $\mathrm{L}$ & 30 & 4.15 & 9.62 & 6.32 & 1.23 & 12 & $6.98^{\mathrm{a}}$ & 1.28 & 18 & $5.88^{\mathrm{a}}$ & 1.01 \\
\hline \multirow{2}{*}{ Kentucky } & $\mathrm{R}$ & 30 & 4.16 & 8.01 & 5.98 & 0.92 & 20 & 6.10 & 0.94 & 10 & 5.75 & 0.87 \\
\hline & $\mathrm{L}$ & 30 & 3.47 & 7.89 & 5.85 & 1.10 & 20 & 5.91 & 1.18 & 10 & 5.72 & 0.99 \\
\hline \multirow{2}{*}{$\begin{array}{l}\text { Africans Americans } \\
\text { Američki crnci }\end{array}$} & $\mathrm{R}$ & 30 & 4.07 & 8.78 & 6.72 & 1.19 & 15 & 7.07 & 1.37 & 15 & 6.38 & 0.89 \\
\hline & $\mathrm{L}$ & 30 & 4.17 & 9.11 & 6.60 & 1.21 & 15 & 7.00 & 1.33 & 15 & 6.20 & 0.96 \\
\hline \multirow{2}{*}{$\begin{array}{l}\text { American Caucasians } \\
\text { Američki bijelci }\end{array}$} & $\mathrm{R}$ & 30 & 4.33 & 9.44 & 6.60 & 1.35 & 15 & $7.19^{b}$ & 1.33 & 15 & $6.00^{\mathrm{b}}$ & 1.13 \\
\hline & $\mathrm{L}$ & 30 & 5.26 & 9.82 & 6.86 & 1.21 & 15 & $7.43^{c}$ & 1.27 & 15 & $6.29^{c}$ & 0.86 \\
\hline
\end{tabular}

$\mathrm{AEH}$ - articular eminence height $\bullet$ visina zglobne kvržice; $\mathrm{N}$ - number of skulls $\bullet$ broj lubanja; Min - minimal value $\bullet$ najmanja vrijednost; Max maximal value $\bullet$ najveća vrijednost; $\mathrm{X}$ - mean value $\bullet$ srednja vrijednost; $\mathrm{SD}$ - standard deviation $\bullet$ standardna devijacija; $\mathrm{R}$ - right $\bullet$ desno; $\mathrm{L}-$ left $\bullet$ lijevo; a,b,c - statistically significant difference according to the sex, $\mathrm{p}<0.05 \bullet$ statistički značajna razlika s obzirom na spol, $\mathrm{p}<0,05$

Table 4 AEL mean values (of all five sections through silicone impressions) for each group according to the body side and sex. Tablica 4. AEL srednje vrijednosti (za svih 5 rezova kroz silikonske otiske) za svaku skupinu s obzirom na stranu tijela i spol

\begin{tabular}{|c|c|c|c|c|c|c|c|c|c|c|c|c|}
\hline \multirow{2}{*}{\multicolumn{2}{|c|}{$\begin{array}{c}\text { AEL } \\
(\mathrm{mm})\end{array}$}} & \multirow{2}{*}{$\mathbf{N}$} & \multirow{2}{*}{ Min } & \multirow{2}{*}{$\begin{array}{l}\text { Max • } \\
\text { Maks. }\end{array}$} & \multirow{2}{*}{$\mathbf{X}$} & \multirow{2}{*}{ SD } & \multicolumn{3}{|c|}{ Men • Muški } & \multicolumn{3}{|c|}{ Women • Ženski } \\
\hline & & & & & & & $\mathrm{N}$ & $\mathrm{X}$ & SD & $\mathbf{N}$ & $\mathrm{X}$ & SD \\
\hline \multirow{2}{*}{ Illinois } & $\mathrm{R}$ & 30 & 9.96 & 14.82 & 11.99 & 1.44 & 12 & 12.59 & 1.63 & 18 & 11.59 & 1.17 \\
\hline & $\mathrm{L}$ & 30 & 9.69 & 16.18 & 11.55 & 1.45 & 12 & $12.37^{\mathrm{a}}$ & 1.75 & 18 & $11.01^{\mathrm{a}}$ & 0.91 \\
\hline \multirow{2}{*}{ Kentucky } & $\mathrm{R}$ & 30 & 9.95 & 13.54 & $12.09^{1}$ & 0.84 & 20 & $12.41^{\mathrm{b}}$ & 0.61 & 10 & $11.43^{b}$ & 0.89 \\
\hline & $\mathrm{L}$ & 30 & 9.44 & 12.92 & $11.57^{1}$ & 1.03 & 20 & 11.80 & 0.91 & 10 & 11.10 & 1.16 \\
\hline \multirow{2}{*}{$\begin{array}{l}\text { Africans Americans } \\
\text { Američki crnci }\end{array}$} & $\mathrm{R}$ & 30 & 9.89 & 14.90 & 12.66 & 1.30 & 15 & 12.93 & 1.26 & 15 & 12.39 & 1.33 \\
\hline & $\mathrm{L}$ & 30 & 10.51 & 15.26 & 12.48 & 1.23 & 15 & $13.02^{c}$ & 1.25 & 15 & $11.94^{c}$ & 0.97 \\
\hline \multirow{2}{*}{$\begin{array}{l}\text { American Caucasians } \\
\text { Američki bijelci }\end{array}$} & $\mathrm{R}$ & 30 & 10.05 & 15.50 & 12.73 & 1.29 & 15 & 12.96 & 1.45 & 15 & 12.50 & 1.10 \\
\hline & $\mathrm{L}$ & 30 & 10.72 & 15.02 & 12.52 & 1.30 & 15 & $12.99^{d}$ & 1.42 & 15 & $12.05^{\mathrm{d}}$ & 1.00 \\
\hline
\end{tabular}

AEL - articular eminence curved line length $\bullet$ duljina zakrivljene linije od najviše do najniže točke na zglobnoj kvržici; $\mathrm{N}$ - number of skulls $\bullet$ broj lubanja; Min - minimal value $\bullet$ najmanja vrijednost; Max - maximal value $\bullet$ najveća vrijednost; $\mathrm{X}$ - mean value $\bullet$ srednja vrijednost; $\mathrm{SD}$ - standard deviation $\bullet$ standardna devijacija; $\mathrm{R}$ - right $\bullet$ desno; $\mathrm{L}$ - left $\bullet$ lijevo; ${ }^{1}$ - statistically significant difference between $\mathrm{R}$ and $\mathrm{L}$ samples, $\mathrm{p}<0.05 \bullet$ statistički značajna razlika između $\mathrm{R}$ i L uzoraka, $\mathrm{p}<0.05$; ${ }^{\mathrm{a}, \mathrm{b}, \mathrm{c}, \mathrm{d}}$ - statistically significant difference according to the sex, $\mathrm{p}<0.05 \bullet$ statistički značajna razlika s obzirom na spol, $\mathrm{p}<0,05$

cone impressions of articular eminence are presented in Table 3.; mean AEL values for all five sections through the silicone impressions of articular eminence are presented in Table 4.

Sex differences in the AEI, AEH, and AEL values were observed, but in most of cases were not statistically significant ce prikazane su u tablici 3.; srednje AEL vrijednosti svih pet rezova kroz silikonske otiske zglobne kvržice prikazane su u tablici 4.

$S$ obzirom na spol, razlike u AEI, AEH i AEL vrijednostima postojale su, ali u većini slučajeva bez statističke zna- 
( $p>0.05$ ), values presented in Tables 1-4.

An analysis of the age groups showed no statistically significant differences $(p>0.05)$ in the AEI, AEH and AEL values for the AAP and ACP populations.

According to the Pearson correlation, the coefficient between AEI M1 and AEI M2; AEI M1 and AEH; AEI M2 and $\mathrm{AEH}$, as well as between AEH and AEL values, all had statistically significant $(\mathrm{p}<0.05)$ and positive correlation (high, with the Pearson correlation coefficient from 0.632 to 0.962 ) regardless of the population. The correlation between the AEI M1 / AEI M2 and AEL values was positive (low or medium, with the Pearson correlation coefficient from 0.116 to 0.485 ) but not statistically significant $(p>0.05)$ regardless of the population.

\section{Discussion}

It has been a well-known fact that the shape and size of craniofacial structures including TMJ is affected by both genetic and environmental factors (19-21, 27). In this context, the aim of this study was to determine the AEI, AEH and AEL values on four ancestrally different American populations along with comparison of measured values between these populations. The AEI, AEH and AEL values presented in this study are means of measured values on all five sections through silicone impressions of the articular eminence. The variability of the articular eminence morphology in anteroposterior and mediolateral direction leads to different $\mathrm{AEI}, \mathrm{AEH}$ and $\mathrm{AEL}$ values from point to point. Considering the fact that the five sections (the sections being $4 \mathrm{~mm}$ apart across the joint) encompasses the whole mediolateral dimension of the articular eminence, the mean values of measured parameters served as a representative result of the measurements. The populations used in this study originated from different geographical locations and time periods. People from the IP sample lived between $900 \mathrm{AD}$ to $1500 \mathrm{AD}$ years alongside the Mississippi River, whereas people from the KP sample lived in the period from $500 \mathrm{BC}$ to $500 \mathrm{AD}$ years alongside the Green River (28). Both populations were usually engaged in fishing as well as in hunting and gathering, but the IP groups were also practicing indigenous agriculture $(28,29)$. The AAP and ACP skulls presented an American contemporary population from the Robert J. Terry Anatomical Collection, thus representing the people living in or around St Louis, Missouri in the first half of the 20th century (30). Due to the shift from foraging / hunting-gathering with harder and tougher food to food production by cooking, providing softer and less tough food (27), it could be assumed that the AEI, AEH and AEL values differ between the prehistoric IP and KP groups, and that there should be a noticeable difference from the prehistoric groups to the contemporary AAP and ACP groups. However, the results obtained by the present study have shown small and statistically nonsignificant differences in the $\mathrm{AEI}, \mathrm{AEH}$ and $\mathrm{AEL}$ values between the investigated populations. But the range of the AEI, $\mathrm{AEH}$ and $\mathrm{AEL}$ values was wide. Mean AEI M1 values ranged from $31.56^{\circ}$ to $38.72^{\circ}$, and the mean AEI M2 values ranged from $44.14^{\circ}$ to $58.37^{\circ}$, regardless of the population group. čajnosti $(\mathrm{p}>0,05)$ - vrijednosti su prikazane u tablicama od 1. do 4 .

$S$ obzirom na dob nema statistički značajnih razlika (p $>0,05)$ u AEI, AEH i AEL vrijednostima za skupine AAP i ACP.

Između AEI M1 i AEI M2; AEI M1 i AEH; AEI M2 i $\mathrm{AEH}$, te između AEH i AEL vrijednosti postoji statistički značajna $(\mathrm{p}<0,05)$ i pozitivna korelacija (visoka, Pearsonov koeficijent korelacije od 0,632 do 0,962) bez obzira na vrstu populacije. Korelacija između AEI M1/AEI M2 i AEL vrijednosti bila je pozitivna (niska ili srednja, $s$ Pearsonovim koeficijentom korelacije od 0,16 do 0,485 ), ali bez statističke značajnosti $(p>0,05)$ bez obzira na vrstu populacije.

\section{Rasprava}

Dobro je poznato da su oblik i veličina kraniofacijalnih struktura, uključujući i temporomandibularni zglob, pod utjecajem genetskih i okolišnih čimbenika $(19-21,27)$. U tom smislu svrha istraživanja bila je odrediti AEI, AEH i AEL vrijednosti na četirima različitim američkim populacijama te ih uzajamno usporediti. AEI, AEH i AEL vrijednosti prikazane u ovom istraživanju srednje su vrijednosti za vrijednosti izmjerene na svih pet rezova kroz silikonske otiske zglobne kvržice. Varijabilnost u morfologiji zglobne kvržice u anteroposteriornom $\mathrm{i}$ laterolateralnom smjeru vodi prema različitim AEI, AEH i AEL vrijednostima izmjerenima od točke do točke. Uzimajući u obzir da pet rezova (razmak između pojedinih rezova je $4 \mathrm{~mm}$ ) obuhvaća cijelu meziodistalnu dimenziju zglobne kvržice, srednje vrijednosti izmjerenih parametara služe kao reprezentativni rezultat mjerenja. Populacije koje su uključene u ovo istraživanje potječu iz različitih geografskih područja i vremenskoga razdoblja. Ljudi iz IP populacije živjeli su između 900. do 1500. godine poslije Krista uzduž rijeke Mississippi, a ljudi iz KP populacije od 500. godine prije Krista do 500. godine poslije Krista uzduž rijeke Green (28). Obje populacije bavile su se ribolovom te lovom i skupljanjem plodova, no IP populacija bavila se i autohtonom poljoprivredom $(28,29)$. AAP i ACP lubanje koje predstavljaju američku suvremenu populaciju dio su anatomske kolekcije Roberta J. Terryja sastavljene od lubanja ljudi koji su živjeli u St. Louisu (Missouri) ili u okolici u prvoj polovici 20. stoljeća (30). Zbog promjene u načinu pronalaženja i pripremi hrane od lova ili skupljanja plodova, što je vezano $s$ tvrđom i žilavijom hranom, na pripremanje hrane kuhanjem, što rezultira mekšom i manje žilavom hranom (27), moglo se pretpostaviti da se AEI, AEH i AEL vrijednosti razlikuju između pretpovijesnih IP i KP populacija i da bi trebala biti uočljiva razlika u vrijednostima istraživanih parametara između pretpovijesnih i suvremenih AAP i ACP populacija. No rezultati istraživanja pokazali su male i statistički neznačajne razlike u AEI, AEH i AEL vrijednostima između istraživanih populacija. Raspon dobivenih AEI, AEH i AEL vrijednosti bio je širok. Srednje AEI M1 vrijednosti bile su u rasponu od 31,56 do $38,72^{\circ}$, a srednje AEI M2 vrijednosti od $44,14^{\circ}$ do $58,37^{\circ}$, bez obzira na populaciju. AEI vrijednosti odraslih obično su u rasponu od $21^{\circ}$ do $64^{\circ}$ (17), što je u skladu s vrijednostima 
The AEI values in adults are usually in range from $21^{\circ}$ to $64^{\circ}$ (17), which corresponds to the values in the present study measured by both, the M1 and M2 method. But, it is often difficult to compare the obtained results with the results from other studies because the authors use different methods and materials for studying the TMJ morphology $(7,9,15$, 19-21). The theory of TMJ change, which is dependent on the type and preparation of the foods in relation with masticatory forces, is not supported by the results obtained in the present study. However, the present study is a scientific contribution to the previously unexplored data of the AEI, AEH and AEL values measured on human skull samples of American prehistoric (IP, KP) and contemporary (AAP and ACP) populations.

The mean AEH values measured in this study were in the range from $5.82 \mathrm{~mm}$ to $6.86 \mathrm{~mm}$ without any statistically significant differences between prehistoric and contemporary American populations. The mean $\mathrm{AEH}$ values from this study are similar to the AEH values measured in other studies although they were not performed on archaeological material (31-34). There is no data about the AEL values found in the literature, but according to the results obtained in this study, the mean AEH values were in range from $11.55 \mathrm{~mm}$ to $12.73 \mathrm{~mm}$ without statistically significant differences between American populations. Koppe et al. (20) measured the sagittal length of temporal articular surface between the postglenoid process and preglenoid plane on human skulls. The sagittal length measured by Koppe et al. (20) was more extended in the anteroposterior direction than the AEL measured in the present study with consequently higher value, which amounted to as high as $26 \mathrm{~mm}$. The results obtained in the present study also indicate a positive correlation between variables describing the articular eminence morphology. In other words, a higher AEI value follows a higher AEH value, and a higher $\mathrm{AEH}$ value results in greater AEL value.

The AEI values were measured by two methods. Both angles (method M1 and M2) represent the measure of AEI (1) although their definitions, and consequently obtained results, are not the same. The AEI values measured by method M2 were statistically significantly higher than AEI values measured by method M1. Similar results were also reported by Katsavrias (1) and Chaurasia et al. (35) with significantly higher AEI values obtained by method M2.

The asymmetry between the left and right side of the face and craniofacial structures (also of the whole body) is a common phenomenon and is usually developed gradually throughout life (36). According to the results obtained in this study, the mean AEI, AEH and AEL values differ between the left and the right TMJ, with difference up to $11^{\circ}$ for the AEI values, up to $1 \mathrm{~mm}$ for the AEH and AEL values, usually without any statistical significance. According to the available literature data $(17,20,21,37)$ and the results of this study, the left-right TMJ differences are a result of natural body asymmetry and possibly different masticatory forces in the left and right TMJ due to the predominant usage of one side during mastication.

The AEI, AEH and AEL values vary between male and female samples with higher values among males but most- dobivenima u ovom istraživanju mjerenjem objema metodama - M1 i M2. No, često je dobivene rezultate teško uspoređivati $s$ rezultatima u drugim istraživanjima jer su se njihovi autori koristili različitim metodama i materijalima $(7,9$, 15, 19 - 21). Teorija o morfološkim promjenama u TMJ-u, ovisno o vrsti hrane i njezinoj pripremi, a povezano sa žvačnim silama, ne može se potkrijepiti rezultatima dobivenima u ovom istraživanju. No znanstveno značenje ovoga istraživanja svakako je u AEI, AEH i AEL vrijednostima koje su dosad bile nepoznate i neistražene na američkim pretpovijesnim (IP, $\mathrm{KP})$ i suvremenim (AAP i ACP) populacijama.

Srednje AEH vrijednosti izmjerene u ovom istraživanju bile su od 5,82 $\mathrm{mm}$ do $6,86 \mathrm{~mm}$, bez statistički značajnih razlika između pretpovijesnih i suvremenih američkih populacija. Srednje AEH vrijednosti dobivene u ovome istraživanju slične su onima iz drugih istraživanja, iako nisu provedene na arheološkom materijalu $(31-34)$. U literaturi nema podataka o AEL vrijednostima, no prema rezultatima ovoga istraživanja srednje AEL vrijednosti bile su u rasponu od $11,55 \mathrm{~mm}$ do $12,73 \mathrm{~mm}$, bez statistički značajnih razlika između američkih populacija. Koppe i suradnici (20) izmjerili su sagitalnu dužinu temporalne artikulacijske površine između postglenoidnog nastavka i preglenoidne ravnine na ljudskim lubanjama. Sagitalna dužina koju su oni izmjerili (20) bila je duža i s većom vrijednošću (do $26 \mathrm{~mm}$ ) u odnosu prema AEL vrijednosti izmjerenoj u ovom istraživanju. Rezultati ovoga istraživanja također upućuju na pozitivnu korelaciju vrijednosti parametara koji opisuju morfologiju zglobne kvržice. Drugim riječima, veće AEI vrijednosti prate veće $\mathrm{AEH}$ vrijednosti, a veće $A E H$ vrijednosti povezane su s većim AEL vrijednostima.

AEI vrijednosti mjerene su dvjema metodama. Oba kuta (metoda M1 i M2) predstavljaju AEI vrijednost (1) iako njihove definicije i posljedično rezultati nisu jednaki. AEI vrijednosti izmjerene metodom M2 statistički su značajno veće od AEI vrijednosti izmjerenih metodom M1. Slične rezultate objavili su i Katsavrias (1) te Chaurasia i suradnici (35) sa značajno većim AEI vrijednostima izmjerenima metodom M2.

Asimetrija između lijeve i desne strane lica i kraniofacijalnih struktura (kao i cijeloga tijela) uobičajena je pojava i nastaje postupno tijekom života (36). Prema rezultatima dobivenima u ovom istraživanju, srednje AEI, AEH i AEL vrijednosti razlikuju se između lijevoga i desnoga TMJ-a, s razlikom do $11^{\circ}$ za AEI vrijednosti, do $1 \mathrm{~mm}$ za AEH i AEL vrijednosti, no najčěsće bez statističke značajnosti. Prema dostupnim rezultatima iz literature $(17,20,21,37)$ te rezultatima iz ovoga istraživanja, razlike između lijevoga i desnoga TMJ-a rezultat su prirodne asimetrije tijela i možda različitih žvačnih sila u lijevom i desnom TMJ-u zbog dominantnog korištenja jedne strane usta pri žvakanju.

AEI, AEH i AEL vrijednosti variraju između muških i ženskih uzoraka $s$ većim vrijednostima među muškarcima, no najčešće bez statističke značajnosti. Podatci iz literature o utjecaju spola na TMJ morfologiju varijabilni su. Wu i suradnici (2012) i Zabarović i suradnici (21) pronašli su spolni dimorfizam sa statistički značajno većim AEI vrijednostima među muškarcima za razliku od žena. Meng i suradnici 
ly without statistical significance. A systematic literature review has shown that the influence of sex on TMJ morphology is variable. Wu et al. (2012) and Zabarović et al. (21) found sexual dimorphism with statistically significantly higher AEI values in males than in females. Meng et al. (15) concluded that there was no statistically significant difference in AEI and $\mathrm{AEH}$ values between males and females.

\section{Conclusion}

The AEI, AEH and AEL values are variable with a wide range of measured values (especially for AEI), but without statistically significant differences between the IP, KP, AAP and ACP groups. It has been previously reported that lifestyle and type of food (masticatory forces) have significant effects on the articular eminence morphology in TMJ morphological change in human evolution. However, the results of the present study neither support the reported conclusions nor confirm the micro evolutionary changes in the TMJ due to the changes in food types or food preparation over the span of the 2,500 years between these groups. This study also found that the body side and sex did not significantly affect the AEI, AEH and AEL values.

\section{Conflict of Interest}

The authors declare that they have no conflict of interest.

Authors' contribution: J.K., D.H. and D.V. - made a research plan. J.K. - made samples and performed measurements. J.K., D.H., D.V., S.P.K., I.K., J.V. - analysed obtained results and participate in writing manuscript.
(15) zaključili su da nema statistički značajnih razlika u AEI i AEH vrijednostima između muškaraca i žena.

\section{Zaključak}

AEI, AEH i AEL vrijednosti varijabilne su $s$ velikim rasponom izmjerenih vrijednosti (posebno AEI-a), ali bez statistički značajnih razlika između skupina IP, KP, AAP i ACP. Poznato je da su način života i vrsta hrane (žvačne sile) tijekom ljudske evolucije imali značajan utjecaj na morfologiju zglobne kvržice. Međutim, rezultati ovoga istraživanja ne podupiru tu tezu, niti potvrđuju mikroevolucijske promjene u TMJ-u zbog promjene u vrsti prehrane i načinu pripreme hrane tijekom razdoblja duljega od 2500 godina. Na temelju rezultata ovoga istraživanja može se zaključiti da strana tijela i spol nisu značajno utjecali na AEI, AEH i AEL vrijednosti.

\section{Sukob interesa}

Autori nisu bili u sukobu interesa.

Doprinos autora: J. K., D. H. i D. V. - pripremili plan istraživanja; J. K. izradio uzorke i obavio mjerenja; J. K., D. H., D. V., S. P. K., I. K., J. V. analizirali dobivene rezultate i sudjelovali u pisanju teksta

\section{Sažetak}

Svrha rada: Istraživanje je provedeno kako bi se dobile vrijednosti nagiba stražnjega zida zglobne kvržice (AEI), visine zglobne kvržice (AEH) i duljine zakrivljene linije od najviše do najniže točke na zglobnoj kvržici (AEL) između američkih povijesnih i suvremenih populacija. Materijal i metode: Istraživanje je obavljeno na 120 ljudskih lubanja podijeljenih u četiri skupine: Illinois (IP), Kentucky (KP), američki crnci (AAP) i američki bijelci (ACP). U svakoj je bilo po 30 lubanja. Mjerenje AEI (dvije metode), AEH i AEL vrijednosti provedeno je s pomoću računalnog programa na pet rezova kroz silikonske otiske zglobne kvržice. Rezultati su statistički obrađeni i analizirani na razini značajnosti od $p<0,05$. Rezultati: Nema statistički značajnih razlika u AEI, AEH i AEL vrijednostima između američkih populacija (IP, KP, AAP, ACP) ni s obzirom na stranu tijela, spol i dob ( $p>0,05)$. Srednje AEI M1 vrijednosti bile su od $31,56^{\circ}$ do $38,72^{\circ}$, srednje AEI M2 vrijednosti od $44,14^{\circ}$ do $58,37^{\circ}$, srednje AEH vrijednosti od 5,82 do $6,86 \mathrm{~mm}$, a srednje AEL vrijednosti od 11,57 do $12,73 \mathrm{~mm}$. Zaključak: AEI, AEH, AEL vrijednosti su varijabilne, ali bez statistički značajnih razlika između američkih populacija. Razlike $s$ obzirom na stranu tijela, kao rezultat prirodne asimetrije tijela, nisu bile statistički značajne. Spol i dob nisu značajno utjecali na morfologiju zglobne kvržice.
Zaprimljen: 10. veljače 2021. Prihvaćen: 21. lipnja 2021.

Adresa za dopisivanje Josip Kranječić Klinički zavod za stomatološku protetiku Klinička bolnica Dubrava Av. G. Šuška 6, 10000 Zagreb, Hrvatska tel: +3851 2902724 faks: +38512864248 kranjcic@sfzg.hr

MeSH terms: disk čeljusnog zgloba; svojstva stanovništva; Afroamerikanci; američki domoroci; preci europskog porijekla

Autorske ključne riječi: lubanja; temporomandibularni zglob; Sjeverna Amerika; populacija

\section{References}

1. Katsavrias EG. Changes in articular eminence inclination during the craniofacial growth period. Angle Orthod. 2002 Jun;72(3):258 64.

2. Kranjcic J, Vojvodic D, Zabarovic D, Vodanovic M, Komar D, Mehulic K. Differences in articular-eminence inclination between medieval and contemporary populations. Arch Oral Biol. 2012 Aug;57(8):1147-52.

3. Kranjcic J, Slaus M, Persic S, Vodanovic M, Vojvodic D. Differences in skeletal components of temporomandibular joint of an ear-
Iy medieval and contemporary Croatian population obtained by a different methods. Ann Anat. 2016 Jan;203:52-8.

4. Okeson PJ. Management of temporomandibular disorders and occlusion. St. Louis:Mosby; 2003.

5. Reicheneder C, Gedrange T, Baumert U, Faltermeier A, Proff P. Variations in inclination of the condylar path in children and adults. Angle Orthod. 2009 Sep;79(5):958-63.

6. Hinton RJ. Changes in articular eminence morphology with dental function. Am J Phys Anthropol. 1981 Apr;54(4):439-55. 
7. Hinton RJ. Relationships between mandibular joint size and craniofacial size in human groups. Arch Oral Biol. 1983;28(1):37-43.

8. Magnusson C, Magnusson T. Size and form of the human temporomandibular joint in African-Americans and Caucasians. Cranio. 2012 Apr;30(2):110-3.

9. Richards LC. Temporomandibular joint morphology in two Australian aboriginal populations. J Dent Res. 1987;66(10):1602-7.

10. Santana ES, Dumont ER, Davis JL. Mechanics of bite force production and its relationship to diet in bats. Funct Ecol. 2010;24(4):776-84.

11. Terhune EC. Dietary correlates of temporomandibular joint morphology in New World primates. J Hum Evol. 2011 Nov;61(5):58396.

12. Vodanovic M, Brkic H, Demo Z, Slaus M. Dental disease and dietary pattern in the early medieval population from Bijelo Brdo East Slavonia, Croatia. Acta Stomatol Croat 2003;37: 386-7.

13. Vodanovic M, Brkic H, Slaus M, Demo Z. The frequency and distribution of caries in the mediaevel population of bijelo Brdo in Croatia (10 th - 11th century). Arch Oral Biol. 2005 Jul;50(7):669-80.

14. Katsavrias EG. The effect of mandibular protrusive (activator) appliances on articular eminence morphology. Angle Orthod. 2003 Dec;73(6):647-53.

15. Meng F, Liu Y, Hu K, Zhao Y, Kong L, Zhou S. A comparative study of the skeletal morphology of the temporomandibular joint of children and adults. J Postgrad Med. Jul-Sep 2008;54(3):191-4.

16. Nickel JC, McLachlan KR, Smith DM. Eminence development of the postnatal human temporomandibular joint. J Dent Res. 1988 Jun;67(6):896-902.

17. Gilboa I, Cardash HS, Kaffe I, Gross MD. Condylar guidance: correlation between articular morphology and panoramic radiographic images in dry human skulls. J Prosthet Dent. 2008 Jun;99(6):477. 82.

18. Seifert D, Jerolimov V, Carek V. Terminal hinge axis-hobo pointFrankfurt horizontal relations. Coll Antropol. 2000 Dec;24(2):47984.

19. Ikai A, Sugisaki M, Young-Sung K, Tanabe H. Morphologic study of the mandibular fossa and the eminence of the temporomandibular joint in relation to the facial structures. Am J Orthod Dentofacial Orthop. 1997 Dec;112(6):634-8.

20. Koppe T, Schöbel SL, Bärenklau M, Bruchhaus H, Jankauskas R, Kaduk WMH. Factors affecting the variation in the adult temporomandibular joint of archaeological human populations. Ann Anat. 2007;189(4):320-5.

21. Zabarovic D, Jerolimov V, Carek V, Vojvodic D, Zabarovic K, Bukovic D. The effect of tooth loss on the TM-joint articular eminence inclination. Coll Antropol. 2000 Jul;24 Suppl 1:37-42.

22. Tanaka E, Koolstra JH. Biomechanics of the temporomandibular joint. J Dent Res. 2008 Nov;87(11):989-91.
23. Owen CP, Wilding RJC, Adams LP. Dimensions of the temporal gle noid fossa and tooth wear in prehistoric human skeletons. Arch Oral Biol. 1992 Jan;37(1):63-7.

24. Badel T, Keros J, Segović S, Komar D. Clinical and tribological view on tooth wear. Acta Stomatol Croat. 2007;41(4):355-65.

25. Celic R, Kraljevic K, Kraljevic S, Badel T, Panduric J. The correlation between temporomandibular disorders and morphological occlusion. Acta Stomatol Croat. 2000;34(1):35-40.

26. Badel T, Panduric J, Marotti M, Krolo I. Clinical investigation of temporomandibular joint arthrosis frequency in young males. Acta Stomatol Croat. 2006;40(1):46-55.

27. Paschetta C, De Azevedo S, Castillo L, Martinez-Abadias N, Hernandez $M$, Lieberman $D E$, et al. The influence of masticatory loading on craniofacial morphology: A test case across technological transitions in the Ohio valley. Am J Phys Anthropol. 2010 Feb;141(2):297-314.

28. Snow EC. Indian knoll skeletons of Site Oh 2, Ohio County, Kentucky. Lexington: University of Kentucky, Department of anthropology; 1948.

29. Titterington PF. Certain bluff mounds of western jersey county, Illinois. Am Antiq. 1935;1(1):6-46.

30. Hunt RD, Albanese J. History and demographic composition of the Robert J. Terry anatomical collection. Am J Phys Anthropol. 2005 Aug;127(4):406-17.

31. Caglayam F, Sümbüllü MA, Akgüul HM. Associations between the articular eminence inclination and condylar bone changes, condylar movements, and condyle and fossa shapes. Oral Radiol. 2014;30:84-91.

32. Okur A, Ozkiris M, Kapusuz Z, Karacavuz S, Saydam L. Characteristics of articular fossa and condyle in patients with temporomandibular joint complaint. Eur Rev Med Pharmacol Sci. 2012 Dec;16(15):2131-5.

33. Ballesteros LE, Ramirez L M, Munoz GM. Mandibular fossa depth variations: relation to age and dental state. Int J Morphol. 2011;29(4):1189-94.

34. Xiang XL, Chen Y, Dai QC, Chen MY. Morphologic survey of temporomandibular joint an autopsy investigation. Shanghai Kou Qiang Yi Xue. 2001 Jun;10(2):142-4.

35. Chaurasia A, Katheriya G, Patil R. Morphometric analysis of articular eminence of temporomandibular joint in Indian Ethinicity - A cone beam computed tomography study. Oral Surg Oral Med Oral Pathol. 1971 Aug;32(2):196-202.

36. Cheong YW, Lo LJ. Facial asymmetry: etiology, evaluation, and management. Chang Gung Med J. Jul-Aug 2011;34(4):341-51.

37. Wu CK, Hsu JT, Shen YW, Chen JH, Shen WC, Fuh LJ. Assessments of inclination of the mandibular fossa by computed tomography in Asian population. Clin Oral Investig. 2012 Apr;16(2):443-50. 